\title{
JOINT FILTERBANK STRUCTURES FOR INTEGRATING AUDIO CODING INTO HEARING AID APPLICATIONS
}

\author{
Rong Dong, David Hermann, Robert Brennan, Edward Chau \\ AMI Semiconductor, 611 Kumpf Drive, Unit 200, Waterloo, Ontario, Canada N2V 1K8
}

\begin{abstract}
To help develop ultra-low power wireless hearing aid products, we investigate the integration of subband audio coding with hearing aid applications. Both the audio coding and the hearing aid application use subband processing, but their requirements for the filterbanks are totally different. The hearing aid application typically uses an oversampled filterbank to reduce the aliasing in each subband, whereas the audio codec needs a critically sampled filterbank for maximal coding efficiency. A joint filterbank structure is proposed in this paper to satisfy these contradictive filterbank requirements. With this structure, the two filterbanks are combined into a single stereo filterbank operation, which can be efficiently implemented on a filterbank coprocessor. This structure substantially reduces the computation complexity, power consumption and memory usage.
\end{abstract}

Index Terms - Channel bank filters, transform coding, audio coding, hearing aids, coprocessors

\section{INTRODUCTION}

Adding wireless functionality to hearing aids brings many benefits to the users [1]. For example, a hearing aid can receive an audio stream from a remote device (e.g., a microphone, television, telephone or another hearing aid on the opposite ear) wirelessly to reduce the negative effects of distance, reverberation and background noise.

In order to reduce the transmission bandwidth, hence the power consumption, for audio streaming, an audio codec is necessary to provide data compression. The audio compression can be performed in either time domain or a subband domain based on time-frequency transforms. However, with subband codec, we can allocate more bits to perceptually important subbands and achieve higher coding gain than the time-domain codecs [2]. Thus, a subband codec is an integral part of the new generation of wireless-capable hearing aids.

However, in a hearing aid device, even though both the codec and the hearing aid algorithms use subband signal processing, their filterbank requirements are very different. The hearing aid algorithms typically apply wide magnitude adjustments in each subband, so they require an oversampled filterbank to achieve a proper tradeoff in terms of aliasing reduction in each subband, group delay, and computation complexity [3]. On the other hand, the subband codec requires a critically sampled filterbank to minimize redundant information and maximize the compression rate. Unfortunately, it is well-known that, in a critically sampled filterbank, the overlapping between adjacent bands generates aliasing distortions that can be cancelled out only if there is little or no modification to the subband signal. This is obviously incompatible with hearing aid signal processing, so challenges arise when we attempt to integrate these two different types of subband processing algorithms. Therefore, in this paper, we investigate and present a novel approach for the efficient integration of a subband codec into a hearing aid application, based on an ultra-low power DSP system including an optimized filterbank coprocessor. Our proposed solution aims for minimizing the computation complexity, power consumption and memory usage.

The rest of the paper is organized as follows. Section 2 describes the architecture of the DSP system and what the coprocessor is capable of. In Section 3, we propose an application framework to solve the problem of the conflicting filterbank requirements. In Section 4 , we propose a selection of filterbanks that are appropriate in this framework, and describe the implementation of the filterbanks using the coprocessor. Finally, Section 5 presents a summary of the paper.

\section{THE DSP SYSTEM}

The DSP system is designed for ultra-low power and miniature audio processing applications. Figure 1 shows a top-level overview of the DSP system. The system is centered around three major digital components: a fixed-point general purpose DSP, a coprocessor and an $\mathrm{I} / \mathrm{O}$ processor. These three components run in parallel and communicate through interrupt signals and shared memory. The general purpose DSP is fully programmable. It provides the flexibility needed to implement a wide range of signal processing algorithms. The coprocessor is specialized for filterbank operations and vector operations. It can perform these operations without intervention from the DSP. In particular, the coprocessor hardware is highly optimized for the implementation of an oversampled complex modulated filterbank. With some adaptation, it can also generate critically-sampled filterbanks [4][5][6]. The filterbank is highly configurable in order to meet the requirements of various applications. It allows for odd or even stacking, different number of subbands, and different type of analysis and synthesis prototype filters. In addition, it can also operate in stereo mode, with two real-valued input signals and a separate prototype filter for each input. With this design, the whole DSP system is particularly efficient for oversampled subband processing. The I/O processor controls the input/output data flow between the input/output stages (i.e., the ADC, DAC and associated circuits) and the FIFO memory. It operates on blocks of data in the background and only interrupts the DSP when necessary. It works in conjunction with multiple I/O channels in the input/output stage concurrently.

Finally, the DSP system also includes clock circuits and a number of other on-chip peripherals. Therefore, it can be easily integrated into miniature devices without many extra external components. A number of analog and digital interfaces are also provided that allow communications with a wide variety of external systems, such as a wireless chip. When used in typical hearing aid applications, the DSP system operates at $1.25 \mathrm{~V}$ supply and consumes less than $1 \mathrm{~mW}$. 


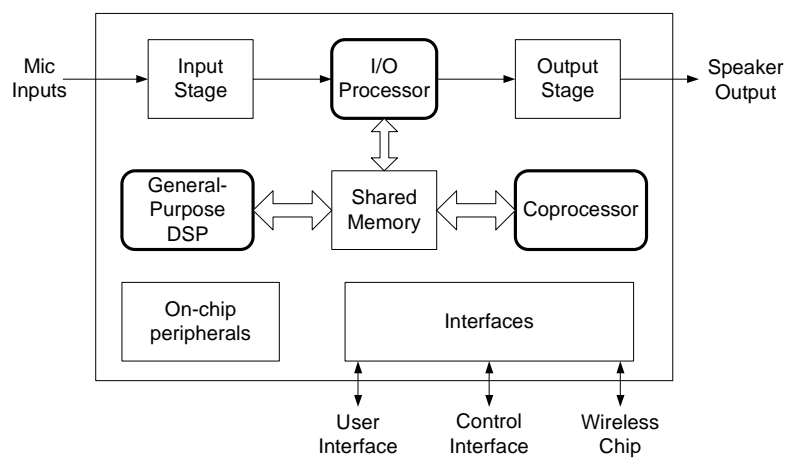

Fig. 1. Block diagram of the DSP system

\section{THE PROPOSED INTEGRATION FRAMEWORK}

In the previous section, we described a DSP system including a specialized coprocessor for efficient implementation of oversampled, complex modulated filterbanks. In this section, we will propose a framework to efficiently deploy the subband codec and the hearing aid application on the DSP system.

As discussed earlier, the codec uses a critically sampled filterbank to maximize the compression rate. The filterbank needs to be carefully designed such that the aliasing components introduced by the downsampling operation in the analysis stage are cancelled out (or approximately cancelled out) in the synthesis stage. However, the critically sampled filterbank is sensitive to subband modification. Any attempt to apply frequency shaping or magnitude adjustments in the subband (which is required in hearing aid signal processing) will lead to severe aliasing distortions in the synthesis output. Therefore, hearing aid processing cannot be performed between the analysis and synthesis of a critically sampled filterbank. In order to solve the conflicting filterbank requirements, we propose a joint filterbank structure using two filterbanks back-to-back.

Figure 2 illustrates the joint filterbank structure in the context of a hearing aid system having a simple wireless audio streaming capability. As shown in the figure, at the transmitter side, the audio input signal is decomposed into a number of subbands through a critically sampled analysis filterbank. The subband signals are then encoded into a bit stream and transmitted over the wireless link. At the receiver side, once a frame of data is received from the wireless link, a decode function is performed to reconstruct the subband signals. Afterwards, the decoded subband signals are transformed back to the time domain using the critically sampled synthesis filterbank. Through this step, the aliased components introduced in the analysis stage are effectively cancelled out by the synthesis filterbank.

Next, the time-domain decoder output is digitally mixed with the local audio input from the receiver side. This can be done in the time domain as shown in Figure 2. The mixed signal is then split into a number of subbands again by an oversampled analysis filterbank. Alternatively, the mixing can also be done in the subband domain. In some hearing aid applications, there may also be other time-domain signal processings performed before the filterbank analysis.

Once the subband mixed signals are available, they are processed by the hearing aid application and then passed through an oversampled synthesis filterbank to generate the time-domain audio output. The time-domain output may also be further processed before being fed into a speaker. Under certain circumstances, the two synthesis filterbanks on the receiver side can be combined into a sin-

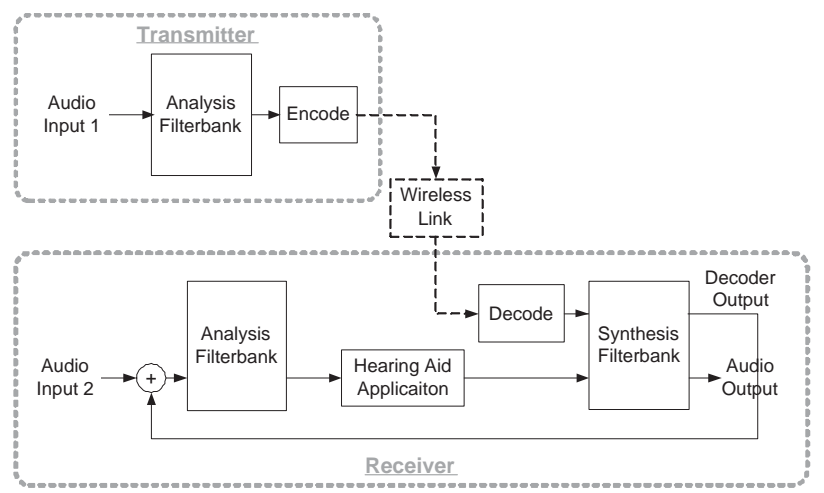

Fig. 2. A proposed framework to integrate a subband codec into a hearing aid application

gle stereo synthesis operation, as explained in the next section. As a result, the clock cycles and memory usage can be reduced substantially by reusing the same operations on the coprocessor.

\section{FILTERBANK SELECTION AND IMPLEMENTATION}

There are two pairs of filterbanks involved in the framework, one for the codec and one for the hearing aid application. Due to the design of the coprocessor, the two filterbanks must have the same stacking configuration (odd or even) and the same number of subbands in order to be combined into a stereo filterbank operation on the coprocessor. Given these constraints, we will propose a selection of filterbanks for the codec that can be used in this framework. The implementation of the filterbanks using the coprocessor will be discussed as well.

\subsection{Complex Modulated Filterbanks for the Hearing Aid Ap- plication}

As explained previously, hearing aid applications require oversampled complex modulated filterbanks (also known as DFT filterbanks). The coprocessor provides an efficient way to implement this type of filterbanks using a weighted overlap-add (WOLA) structure [3][7]. A block diagram of a single subband channel in an oversampled complex modulated filterbank is shown in Figure 3. Essentially, the subband filters are obtained through the complex modulation of a low-pass prototype filter. The impulse response of the analysis and synthesis filters can be expressed by

$$
\begin{aligned}
& h_{k}^{D F T}(n)=h(n) e^{j \omega_{k} n} \\
& g_{k}^{D F T}(n)=g(n) e^{j \omega_{k} n}
\end{aligned}
$$

For odd-stacking filterbank, each subband filter is located at center frequency $\omega_{k}=\frac{\pi}{M}\left(k+\frac{1}{2}\right)$ with $k=0, \ldots, 2 M-1$. As a result, an odd-stacking filterbank produces a total number of $2 M$ subbands. For even-stacking filterbank, the center frequency is located at $\omega_{k}=$ $\frac{\pi}{M} k$ with $k=0, \ldots, 2 M$. As a result, an even-stacking filterbank produces a total number of $2 M+1$ subbands including the bands centered at the DC and Nyquist frequency. But, in typical audio applications, only half of the bands are unique because of the realvalued input signal and prototype filter coefficients.

In the analysis stage, the time domain signal $x(n)$ is decomposed into subband signals by the subband analysis filters defined in (1). The subband signals are decimated by a factor of $R(R \leq M)$. 

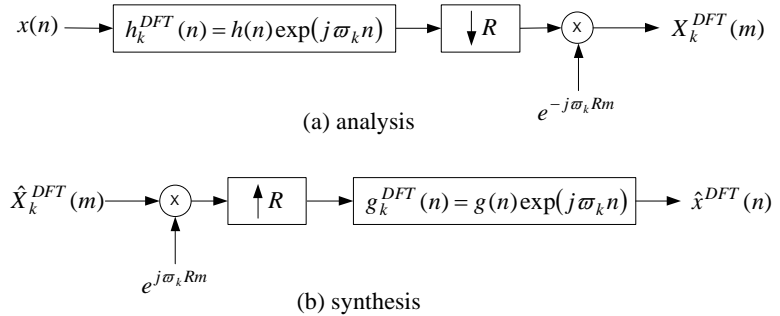

Fig. 3. Single channel of a complex modulated filterbank

The decimated signals are modulated by a function of $e^{-j \omega_{k} R m}$ so that the center of the band is shifted to DC. The resulting decimated subband signals are denoted as $X_{k}^{D F T}(m)$. In the synthesis stage, the input subband signals $\hat{X}_{k}^{D F T}(m)$ are shifted back to the original frequency locations and then interpolated by a factor of $R$. The imaging components generated by the interpolation are filtered out by the subband synthesis filters defined in (1). The resulting subband signals are then recombined into a time-domain output signal.

\subsection{Odd-Stacking Critically Sampled Filterbanks for the Codec}

If the hearing aid application uses an odd-stacking filterbank, the codec must also use an odd-stacking critically sampled filterbank so that the two filterbanks can be easily combined into one stereo filterbank operation. In this case, a critically sampled cosine modulated filterbank is a good choice for reasons of coding efficiency and simplified processing.

In a cosine modulated filterbank, all the subband filters are derived from a prototype filter by cosine modulation. In contrast to the complex modulated filterbank, the cosine modulated filterbank produces real-valued subband samples for a real-valued input signal. This is a desirable feature in coding applications. The block diagram of a typical critically sampled odd stacking cosine modulated filterbank is similar to the block diagram of a complex modulated filterbank. For audio coding, the cosine modulated filterbank does not use the frequency shift $e^{ \pm j \omega_{k} R m}$ [8]. For critical sampling, the decimation factor $R$ must be equal to the number of subbands $M$. If not, we can further decimate the subband signals by a factor of $M / R$ in order to achieve critical sampling.

A variety of cosine modulated filterbanks have been studied for audio codec applications. One of the most common variants is cosine modulated pseudo-QMF filterbank [8]. The analysis and synthesis filters of the cosine modulated pseudo-QMF filterbank are given by

$$
\begin{aligned}
& h_{k}^{C M F B}(n)=2 p(n) \cos \left[\omega_{k}\left(n-\frac{L}{2}\right)+(-1)^{k} \frac{\pi}{4}\right] \\
& g_{k}^{C M F B}(n)=2 p(n) \cos \left[\omega_{k}\left(n-\frac{L}{2}\right)-(-1)^{k} \frac{\pi}{4}\right]
\end{aligned}
$$

Each subband filter is located at center frequency $\omega_{k}=\frac{\pi}{M}\left(k+\frac{1}{2}\right)$ with $k=0, \ldots, M-1$. In (2), $L$ is the length of the prototype filter $p(n)$. The phase term $\pm(-1)^{k} \frac{\pi}{4}$ is chosen to eliminate the phase distortion and approximately cancel the alias distortion.

Another important class of cosine modulated filterbanks is based on lapped transforms. A lapped transform is essentially a critically sampled cosine modulated filterbank that has the perfect reconstruction (PR) property. The equivalent analysis and synthesis filters of the lapped transform are defined by [9]

$$
\begin{aligned}
& h_{k}^{L T}(n)=\sqrt{\frac{2}{M}} p(n) \cos \left[\omega_{k}\left(n+\frac{1-M}{2}\right)\right] \\
& g_{k}^{L T}(n)=\sqrt{\frac{2}{M}} p(n) \cos \left[\omega_{k}\left(n+\frac{1+M}{2}\right)\right]
\end{aligned}
$$

Again, each subband filter is located at center frequency $\omega_{k}=\frac{\pi}{M}(k+$ $\left.\frac{1}{2}\right)$ with $k=0, \ldots, M-1$. If the length of the prototype filter $p(n)$ is $L=2 M$, the transformation is known as modified discrete cosine transform (MDCT), or lapped orthogonal transform (LOT). If a longer prototype filter is used $(L>2 M)$, then the transformation is known as extended lapped transform (ELT). In either cases, the prototype filter must be designed carefully to achieve perfect reconstruction. Some design examples are presented in [9].

Although the coprocessor in our DSP system is designed for complex modulated filterbanks based on the WOLA approach, our previous works have shown that it is possible to implement cosine modulated filterbanks using the coprocessor. For example, the method to implement the filterbank used by the Bluetooth subband codec is presented in [4]. Later work in [6] derives the conversion from a complex modulated filterbank to a cosine modulated pseudo QMF filterbank. In this work, we will combine these ideas and extend them to a universal framework for implementing various forms of cosine modulated filterbanks using a WOLA filterbank coprocessor.

In order to derive a universal solution, let us rewrite the general form of cosine modulated filters as follows

$$
\begin{aligned}
& h_{k}^{C O S}(n)=\alpha h(n) \cos \left(\omega_{k} n+\varphi_{k}^{(A)}\right) \\
& g_{k}^{C O S}(n)=\beta g(n) \cos \left(\omega_{k} n+\varphi_{k}^{(S)}\right)
\end{aligned}
$$

where $\alpha$ and $\beta$ are the real-valued constant scaling factors; $\varphi_{k}^{(A)}$ and $\varphi_{k}^{(S)}$ are the frequency-dependent phase terms. Different definition of the phase terms and the constant scaling factors leads to a specific form of cosine modulated filterbanks. The cosine modulated pseudoQMF filterbank defined in (2) and the lapped transform defined in (3) are just two special cases of (4).

By comparing the definition in (1) and (4), the subband output of the cosine modulated analysis filters $X_{k}^{C O S}(m)$ can be expressed using the subband output of the complex modulated analysis filters $X_{k}^{D F T}(m)$, assuming the same prototype filter:

$$
\begin{gathered}
X_{k}^{C O S}(m)=\alpha R e\left\{X_{k}^{D F T}(m) e^{j \omega_{k} M m} e^{j \varphi_{k}^{(A)}}\right\} \\
k=0, \ldots, M-1
\end{gathered}
$$

It takes three steps to convert the oversampled complex modulated filterbank output to the equivalent critically sampled cosine modulated filterbank output. The first step is to apply a phase shift and a scaling factor $\alpha e^{j \varphi_{k}^{(A)}}$. Since this part is constant over time, we can pre-store the values in a complex vector and apply it via a complex gain application operation available on the coprocessor. The second step is to multiply a demodulation factor $e^{j \omega_{k} M m}$. The value of the demodulation factor varies over the subband index $k$ and the block index $m$. As the coprocessor is not designed for this type of operations, the demodulation will need to be done on the general DSP. Finally, the last step is taking the real part of the resulting complex subband samples. Note that the demodulation factor has a simple repeating pattern composed of \pm 1 and $\pm j$ [4]. Therefore, the demodulation along with the extraction of the real parts can be simply done by taking or negating the real and imaginary parts of the subband samples in an repeatable pattern.

Likewise, for synthesis, it is possible to use the complex modulated synthesis filterbank to reconstruct a time-domain signal that is exactly the same as the one reconstructed by the cosine modulated filterbank. In order to do that, the following pre-transformation is applied on the subband input signals $\hat{X}_{k}^{C O S}(m)$.

$$
\begin{array}{ll}
\hat{X}_{k}^{D F T}(m)=\frac{\beta}{2} \hat{X}_{k}^{C O S}(m) e^{-j \omega_{k} M m} e^{j \varphi_{k}^{(S)}}, k=0, \ldots, M-1 \\
\hat{X}_{k}^{D F T}(m)=X_{2 M-1-k}^{D F T}(m)^{*}, & k=M, \ldots, 2 M-1
\end{array}
$$




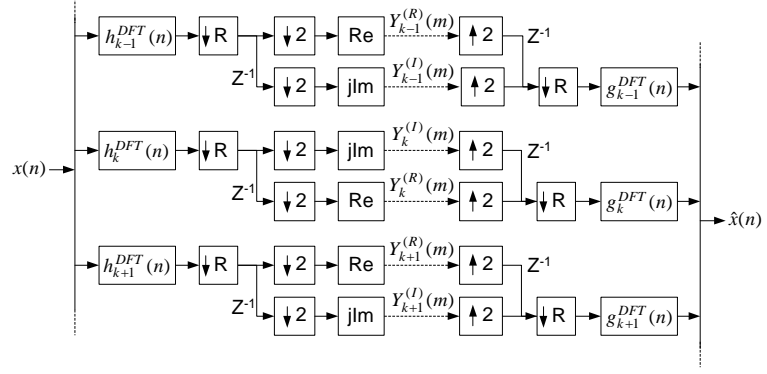

Fig. 4. Modified DFT filterbank

The resulting subband signals $\hat{X}_{k}^{D F T}(m)$ is then fed into the complex modulated synthesis filterbank. The conversion for the synthesis filterbank can be done in two steps: the first step is to rebuild the complex subband samples by applying the modulation factor $e^{-j \omega_{k} M m}$. Again, due to the repeating pattern of \pm 1 and $\pm j$, the modulation is essentially taking or negating the real subband samples and then filling them into the real or imaginary spots. This step can be easily done on the general DSP. The second step is to apply a phase shift and a scaling factor $\frac{\beta}{2} e^{j \varphi_{k}^{(S)}}$ via a complex gain application operation on the coprocessor. The higher frequency bands are symmetric complex conjugate of the lower frequency bands. The conjugation is automatically taken care of by the hardware.

\subsection{Even-Stacking Critically Sampled Filterbanks for the Codec}

Similar to the odd-stacking case, if the hearing aid application uses an even-stacking filterbank, the codec must also use an even-stacking critically sampled filterbank. In this case, a critically sampled modified DFT (MDFT) filterbank is a good choice for the codec.

Modified DFT (MDFT) filterbank is a critically sampled complex modulated filterbank [10]. It can provide perfect reconstruction by a proper design of the prototype filter. The detailed structure of the filterbank is shown in Figure 4. Same as the definition in (1), the analysis and synthesis filters are obtained from the prototype filter through complex modulation. Each subband signal is decimated by a factor of $R$. For critical sampling, $R=M$. If not, we can further decimate the subband signals by a factor of $M / R$. After that, each complex subband signal is converted to a real subband signal by taking only the real or the imaginary part at a time. On the synthesis side, the subband signals are reconstructed from the real or the imaginary part and then processed by the synthesis filterbank.

Therefore, a modified DFT (MDFT) filterbank is essentially an oversampled complex modulated filterbank with additional decimation and interpolation in the middle. The oversampled complex modulated filterbank can be easily and efficiently converted to/from a MDFT filterbank, combining with the stereo filterbank operation available on the coprocessor. The additional decimation and interpolation is simply taking real and imaginary part of the complex subband samples, which can be easily done on the general DSP.

\section{CONCLUSION}

In this paper, we have described a DSP system that was specially designed for ultra-low power audio processing. The system contains a coprocessor for efficient implementation of oversampled complex modulated filterbanks. In order to enable low-power digital wireless audio streaming in hearing aid devices, we have presented an efficient approach to integrate subband audio coding with hearing aid signal processing on this DSP system. Due to conflicting filterbank requirements by these two types of signal processing, a joint filterbank structure is presented in this paper. It allows us to convert between different types of filterbanks using the same coprocessor originally designed for oversampled filterbanks. Depending on the configuration of the filterbank for the hearing aid application, we have also proposed a selection of compatible filterbanks for the codec that can be fitted into this joint filterbank structure. If the hearing aid application uses an odd-stacking filterbank, the codec can use a critically sampled cosine modulated filterbank. Similarly with an even-stacking filterbank, the codec can use a critically sampled modified DFT filterbank. To facilitate conversion between the different types of filterbanks using the filterbank coprocessor, we have derived a universal formula for converting an oversampled complex modulated filterbank to various forms of critically sampled cosine modulated filterbanks. With the joint filterbank structure, we can use a single stereo oversampled complex modulated filterbank and a simple conversion to implement two different types of filterbanks concurrently, which is computationally less expensive than implementing two filterbanks separately.

\section{REFERENCES}

[1] A. Boothroyd, K. Fitz, J. Kindred, S. Kochkin, H. Levitt, B.C. Moore, and J. Yanz, "Hearing aids and wireless technology," The Hearing Review, June 2007.

[2] N.S. Jayant and P. Noll, Digital Coding of Waveforms, Prentice Hall, 1984.

[3] R. Brennan and T. Schneider, "Flexible filterbank structure for extensive signal manipulations in digital hearing aids," in Proceedings of the IEEE International Symposium on Circuits and Systems (ISCAS 1998), Monterey, CA, USA, May 1998, vol. 6, pp. 569-572.

[4] D. Hermann, R. L .Brennan, H. Sheikhzadeh, and E. Cornu, "Low-power implementation of the Bluetooth subband audio codec," in Proceedings of the IEEE International Conference on Acoustics, Speech and Signal Processing (ICASSP 2004), Montreal, Canada, May 2004.

[5] J. Johnson, D. Hermann, M. Witter, E. Cornu, R. Brennan, and A. Dufaux, "An ultra-low power subband-based electronic stethoscope," in Proceedings of the IEEE International Conference on Acoustics, Speech and Signal Processing (ICASSP 2006), Toulouse, France, May 2006.

[6] E. Cornu, A. Dufaux, and D. Hermann, "A high performance, low latency, low power audio processing system for wideband speech over wireless links," in Proceedings of the European Signal Processing Conference (EUSIPCO 2006), Florence, Italy, September 2006.

[7] R.E. Crochiere and L.R. Rabiner, Multirate Digital Signal Processing, Prentice Hall, 1983.

[8] P.P. Vaidyanathan, Multirate Systems and Filter Banks, Prentice Hall, 1993.

[9] H. S. Malvar, Signal Processing with Lapped Transforms, Artech House, 1992.

[10] T. Karp and N. J. Fliege, "Modified DFT filter banks with perfect reconstruction," IEEE Transactions on Circuits and Systems II: Analog and Digital Signal Processing, vol. 46, no. 11, pp. 1404-1414, November 1999. 